\section{Global Journal of Psychology Research: New Trends and Issues}

Volume 06, Issue 4, (2016) 185-194
Global Journal of Psychology Research: New Trends and Issues

\title{
Stress factors and quality of life in adult hemodialysis patients
}

\author{
Camelia Soponaru", Faculty of Psychology and Education Sciences Medical Psychology and Special \\ Psychopedagogy, "Al. I. Cuza" University, Str. Toma Cozma, nr. 3, Iaşi, 700554 lasi, Romania. \\ Ancuta Bojian, Department of Psychology, Faculty of Psychology and Educational Sciences, University "Al.I. \\ Cuza" of lasi, Romania. \\ Magdalena lorga, Department of Behavioral Sciences, University of Medicine and Pharmacy "Gr.T. Popa" of lasi, \\ "St. Maria" Child Hospital, lasi, Romania.
}

\section{Suggested Citation:}

Soponaru, C., Bojian, A., \& lorga, M. (2016). Stress factors and quality of life in adult hemodialysis patients. Global Journal of Psychology Research: New Trends and Issues. 6(4), 185-194.

Received September 18, 2016; revised October 23, 2016 ; accepted November 30, 2016;

Selection and peer review under responsibility of Prof. Dr. Tulay Bozkurt, Istanbul Kultur University, Turkey.

${ }^{\circ} 2016$ SciencePark Research, Organization \& Counseling. All rights reserved.

\begin{abstract}
The aim of the study is to investigate the influence of physiological and psychosocial stress on the quality of life (QoL) in a group of hemodialysis (HD) patients from our center. Materials and methods: A number of 70 patients were included in the study. We used the Hemodialysis Stressors Scale, in order to estimate the stress level, and the Short Form-36 (SF-36) Health Survey Questionnaire, to evaluate the QoL. Results: The $M$ for age $=54.5 \pm 11.7$ years and 38.6\% were men. Patients with a low level of stress had significantly higher physical health $(M=53,52 ; p=0.04)$ and mental health $(M=51,61 ; p<0.01)$ scores. The disease-related factors that induced the highest level of stress were the physical symptoms $(1.67 \pm 0.68)$, the food and water intake restrictions $(1.64 \pm 0.76)$, the vascular access problems $(1.44 \pm 0.83)$, the daily activity limitations $(1.22 \pm 0.72)$, the dependence on the medical staff $(1.14 \pm 0.74)$. Socio-economical and demographical factors had no effect on stress and QoL. Conclusions: The study prove the importance of stress for the QoL in HD patients. Efforts should be undertaken to minimize stress factors.
\end{abstract}

Keywords: dialysis, stress, physical well-being, psychological well-being, quality of life

* ADDRESS FOR CORRESPONDENCE: Camelia Soponaru, Faculty of Psychology and Education Sciences Medical Psychology and Special Psychopedagogy, "Al. I. Cuza" University, Str. Toma Cozma, nr. 3, Iaşi, 700554 lasi, Romania.E-mail address:puzdriac@yahoo.com 


\section{Introduction}

End-stage kidney disease is a long-term debilitating, incurable medical condition, which requires as forms of treatment dialysis or kidney transplantation. The most frequently encountered means of life support for the chronic kidney patient is hemodialysis. What makes this chronic disease unique is the fact that death can be postponed only by following this invasive treatment. Patients confront their mortality each time they come in for the hemodialysis procedure, and their dependence on the artificial kidney permanently reminds them of the vulnerable state they are in.

Hemodialysis is almost automatically associated with numerous stressors which may significantly decrease patients' overall quality of life, finding out about such a diagnosis being a stressful event in itself; but without this form of treatment, survival would no longer be possible. The price of prolonging life by means of the treatment is paid by the patient over time, by restrictions, fatigability, limited social roles and leisure, as well as, in some cases, giving up one's professional status.

Despite the increased prevalence of patients suffering from CKD in Romania and of centers providing healthcare services for them, more accurately, despite the awareness of the impact of CKD on the population, we could not identify any scientific studies carried out in order to investigate the level of stress and its effects on quality of life among this specific group of patients, or the specific factors ranked in the top positions of the hierarchy of factors generating HD patients' high level of stress. Nonetheless, in a study on Romanian patients, data showed that HD patients have a low quality of life, by comparison with the general population, and that at least one third of them should follow rehabilitation therapy. Beside the fact that, in the last 20 years in Romania, like in other Central and Eastern European countries, an expansion in the number of dialysis centers has taken place, there has also been an endeavor aimed at improving these patients' quality of life, that is, the addition of a psychologist to the team of specialists. In addition, Romanian specialists have validated two instruments for the assessment of HD patients' quality of life, also offering a set of indications about psychological practice in this field (Seica, Segall, Verzan, Vaduva, Madincea, Rusoiu \& Grajdeanu, 2009). This study aims to continue the ongoing endeavors regarding the study of factors influencing HD patients' quality of life, focusing on stressors.

Such information may help specialists develop and implement strategies for improving patients' quality of life, but it may also be transmitted to patients, in order to maximize their level of comprehension of their own situation and experience. A better understanding of the disease is associated with high control over it and with a lower emotional response (Covic, Seica, GusbethTatomir, Gavrilovici \& Goldsmith, 2004).

The objectives of research are the following:

1. Studying the effect that the level of stress experienced by hemodialysis patients has on their quality of life. By extension, we will identify the effect stress exerts at the level of hemodialysis patients' physical and mental health.

2. Ranking hemodialytic stressors according to the intensity with which their influence is felt by patients.

3. Identifying significant predictors, among the seven stressors, for quality of life, physical and mental health.

4. Identifying the differences in what concerns the stress felt by hemodialysis patients and quality of life according to the demographic variables gender, original environment, marital status and level of education. 


\section{Material and methods}

The research group consists of patients diagnosed with stage 5 chronic kidney disease, who undergo hemodialysis as a form of treatment, at a dialysis center in North-Eastern Romania. A total of 70 patients voluntarily participated in the study. Of these, $38.6 \%$ were male and $61.4 \%$ were female. The subjects of this research were aged from 19 to 76, the mean age being $54.55(S D=11.70)$. Of the $70 \mathrm{HD}$ patients, $52.9 \%$ live in an urban environment, while $42.9 \%$ live in a rural environment. Analyzing subjects' level of education, we could note that $14.3 \%$ of them had at most 8 years of schooling, $54.9 \%$ graduated from 9 to 12 grades and $30 \%$ had over 12 years of schooling. In addition, we were interested in identifying subjects' marital status, and we could thus establish that $14.3 \%$ were single, while $85.7 \%$ were involved in a relationship.

The selection of participants was based on a number of criteria, such as: having started the hemodialytic treatment of renal function replacement at least 6 months before, being at least 18 years old and having the cognitive ability to understand the meaning of items of the research instruments and giving an answer according to their subjective feelings.

The sampling method used was for convenience, non-random, being justified by subjects' specific features, but also by the researcher's resources. The process of data collection took place from March 2014 to December 2014. Patients were given an informed consent, by which they were made aware of the purpose of the research, the instructions for filling in the instruments used throughout the study, the principles of data privacy, as well as the possibility to withdraw at any moment from the group of subjects, without repercussions of any kind, especially those connected to the quality of health services they benefit from.

There were two ways to fill in the instruments: as an interview conducted by the researcher, or by self-administration. In the first situation, filling in the surveys took place during hemodialysis sessions, while the self-administration of instruments entailed their filling in by subjects at home, after being given instructions for it. Demographic data such as: age, gender, marital status, level of education and original environment were taken into consideration.

Two instruments were used for the research: the Hemodialysis Stressor Scale (in order to check the internal consistency of this scale, the Alpha Cronbach coefficient was calculated both for the entire instrument - Alpha Cronbach $=0.915$ and for each of the seven dimensions) and The 36-Item Short Form Survey (SF-36) validated Romanian Version (the internal consistency of this instrument was proved by calculating the Alpha Cronbach coefficient with Alpha Cronbach $=0,82$ ).

The testing of the four main objectives of this study was performed by means of statistical operations carried out by the SPSS 17.00 software for statistical analysis.

In order to identify the extent to which the level of stress (high vs. low) influences quality of life in hemodialysis patients, we applied the t test for independent samples. To check the extent to which hemodialysis patients' physical and mental health differs according to the level of stress that they feel, the same t test for independent samples was used. In order to clarify the descending hierarchy of intensity of the stressors which exert their influence on HD patients, the arithmetic mean was calculated for each scale of the HSS instrument and, afterwards, we found the mean and standard deviation of the new variables. The significant predictors of quality of life, physical and mental health were established by means of a simple linear regression. The effects of the variables gender, marital status and original environment were tested by the $t$ test for independent samples, while the differences according to the level of education were identified by applying the One-Way ANOVA method. 


\section{Results}

Subjects with a low level of stress obtained a mean equal to 108.66 in the quality of life variable, whereas the mean of subjects with a high level of stress in the quality of life variable was 97.70 . Given that $t(29)=2.59$ and $p=0.01<0.05$, the difference between the two means turns out to be significant, therefore we may state that subjects with a low level of stress have a significantly better quality of life, compared to those with a high level of stress. These results are shown in the Table 1:

Table 1. Results of the t test for comparing means in the quality of life, physical health and mental health

\begin{tabular}{llllllll}
\multicolumn{7}{c}{ variables according to the stress variable } \\
\hline Variables & $\mathrm{N}$ & $\mathrm{M}$ & $\mathrm{SD}$ & $\mathrm{t}$ & $\mathrm{df}$ & $\mathrm{p}$ \\
\hline $\begin{array}{l}\text { Quality of life } \\
\text { Low stress }\end{array}$ & 21 & 108.66 & 12.80 & & & \\
$\begin{array}{l}\text { High stress } \\
\text { Physical }\end{array}$ & 10 & 97.70 & 10.04 & & & \\
health & & & & 2.145 & 29 & 0.040 \\
Low stress & 21 & 53.52 & 7.20 & & & \\
$\begin{array}{l}\text { High stress } \\
\text { Mental stress }\end{array}$ & 10 & 47.90 & 5.89 & & & \\
Low stress & 21 & 51.61 & 6.68 & & & \\
High stress & 10 & 44.60 & 5.12 & & & \\
\hline
\end{tabular}

In addition, there are significant differences, according to the level of stress, in what concerns subjects' physical health [t $(29)=2.145, p=0.040]$. Thus, patients with a low level of stress obtain, on average, significantly higher scores in the quality of life variable $(M 1=53.52)$, compared to patients whose level of stress is high ( $\mathrm{M} 2=47.90)$.

Last, but not least, the results reveal the existence of statistically significant differences [t $(29)=$ $2.925, p=0.007$ ] between the means obtained in the mental health variable by subjects with a low level of stress ( $M 1=51.61)$, compared to subjects whose recorded level of stress is high $(M 2=44.60)$.

The hemodialytic factor exerting the highest level of stress on patients is represented by the physical symptoms they confront $(M=1.67, S D=0.68)$, followed by food and fluid restrictions $(M=$ $1.64, S D=0.76)$, vascular access $(M=1.44, S D=0.83)$, daily activity $(M=1.22, S D=0.72)$, dependence on medical staff $(M=1.14, S D=0.74)$, role ambiguity $(M=0.99, S D=0.86)$, the functioning of the reproductive system $(M=0.77, S D=0.69)$.

For a more thorough analysis, we set out to rank the 31 items, which represent, in fact, a particular stressor, according to the assessment of the 70 subjects of this research. Thus, the mean was calculated for all 31 items. Analyzing also the following data of the research, we noticed that 9 of the first 10 items identified as the most stressful are of a physiological nature, finding one single item of a psychological nature, that is, "the uncertainty of the future". Item 9, "Fatigue", recorded a mean of 2.00 (SD = 0.85), being followed by "Painful joints" $(M=1.98, S D=1.02)$, "Muscle cramps" $(M=1.85$, $S D=1.09)$, "Fluid restrictions" $(M=1.85, S D=1.13)$, etc. In this study, the items ranked in the last three positions, by mean, are: "Transport to/from the clinic" ( $M=0.75, D S=0.96)$, "Decreased sex drive" ( $M=0.74$, DS = 0.92), "Role reversal with one's children" ( $M=0.68, D S=0.98)$.

In order to identify significant predictors of quality of life, seven simple linear regressions were carried out. The results obtained for the group of subjects $(N=70)$ who took part in this study 
highlight the fact that the predictor explaining most of the variance of the quality of life criterion is dependence on medical staff (18.7\%), followed by daily activity (13.9\%), food and fluid restrictions (7.7\%) and role ambiguity (7.4\%). An important mention is that all four significant predictors of quality of life are negative (see Table 2 ).

Table 2. Results of the regression analysis concerning the estimation of quality of life based on the variables

\begin{tabular}{llllllll}
\hline Variables & $\mathrm{R}$ & $\mathrm{R}^{2}$ & $\beta$ & $\mathrm{b}$ & $\mathrm{SEb}$ & $\mathrm{F}$ & $\mathrm{p}$ \\
\hline Daily activity & 0.372 & 0.139 & -0.372 & -0.948 & 0.287 & 10.936 & 0.002 \\
Physical symptoms & 0.204 & 0.042 & -0.204 & -0.474 & 0.276 & 2.955 & 0.090 \\
Dependence on medical staff & 0.433 & 0.187 & -0.433 & -1.281 & 0.324 & 15.665 & 0.000 \\
Food and fluid restrictions & 0.277 & 0.077 & -0.277 & -1.004 & 0.423 & 5.644 & 0.020 \\
Role ambiguity & 0.272 & 0.074 & -0.272 & -1.156 & 0.497 & 5.416 & 0.023 \\
Vascular access & 0.169 & 0.029 & -0.169 & -0.751 & 0.531 & 2.000 & 0.162 \\
Functioning of the reproductive system & 0.221 & 0.049 & -0.221 & -1.171 & 0.628 & 3.483 & 0.066 \\
\hline
\end{tabular}

For physical health, one of the two dimensions of quality of life, the significant, negative predictors were dependence on medical staff $(9.7 \%)$ and daily activity (8.7\%). Meanwhile, for mental health, the following stressors were identified as significant predictors: dependence on medical staff $(25.3 \%)$, daily activity (16.4\%), food and fluid restrictions $(13.4 \%)$, physical symptoms $(10.9 \%)$ and role ambiguity (10.3\%). This time, too, the predictors are negative. The summarized statistical data are shown in Table 3, but their interpretation must be carried out with caution, taking into account the number of subjects who participated in this research, but also their specific features.

Table 3. Results of the regression analysis concerning the estimation of physical and mental health based on the

\begin{tabular}{|c|c|c|c|c|c|c|c|}
\hline \multicolumn{8}{|c|}{ ables } \\
\hline Variables & $R$ & $\mathrm{R}^{2}$ & $\beta$ & $\mathrm{b}$ & SEb & $\mathrm{F}$ & $p$ \\
\hline \multicolumn{8}{|l|}{ Physical health } \\
\hline Daily activity & 0.294 & 0.087 & -0.294 & -0.433 & 0.171 & 6.446 & 0.013 \\
\hline $\begin{array}{l}\text { Dependence on medical staff } \\
\text { Mental health }\end{array}$ & 0.312 & 0.097 & -0.312 & -0.533 & 0.197 & 7.312 & 0.009 \\
\hline Daily activity & 0.405 & 0.164 & -0.405 & -0.562 & 0.154 & 13.368 & 0.000 \\
\hline Physical symptoms & 0.331 & 0.109 & -0.331 & -0.418 & 0.145 & 8.351 & 0.005 \\
\hline Dependence on medical staff & 0.503 & 0.253 & -0.503 & -0.811 & 0.169 & 23.058 & 0.000 \\
\hline Food and fluid restrictions & 0.366 & 0.134 & -0.366 & -0.722 & 0.223 & 10.508 & 0.002 \\
\hline Role ambiguity & 0.321 & 0.103 & -0.321 & -0.744 & 0.266 & 7.812 & 0.007 \\
\hline
\end{tabular}

In order to test the effect of the demographic variables gender, marital status and original environment on the stress felt by hemodialysis patients, we used the method of the test for independent samples, while the effect of the variable level of education was tested by means of the One-Way ANOVA method. Male subjects obtained a mean of 39.03 in the stress variable, while female subjects obtained a mean of 42.34 in the same dependent variable. However, the difference between the two means is not a significant one [ $\mathrm{t}(68)=-0.77, \mathrm{p}=0.44$ ]. Looking into the effect of marital status on the stress variable, we analyzed, more specifically, whether there were differences between the scores obtained in the stress variable by subjects involved in a relationship, compared to subjects who were single. The mean obtained in the stress variable by single subjects was 42.90 , while subjects who were in a relationship obtained a mean equal to 40.76 . We may notice that single subjects have a 
higher level of stress, but this difference is not statistically significant [t $(68)=0.35, p=0.72$ ]. Subjects with less than 8 years of schooling recorded a mean of 36.50 in the stress variable, those with 9 to 12 years of schooling had a mean of 40.13 , and those with over 12 years of schooling had a mean equal to 54.14. Since $F(2,65)=0.951$, and the value of coefficient $p$ is equal to 0.392 , we conclude that there is no significant effect of the variable level of education on the stress felt by hemodialysis patients. All these data are summarized in the Table 4:

Table 4. Statistical results obtained from testing the effect of the demographic variables on stress and quality of

\begin{tabular}{|c|c|c|c|c|}
\hline Variables & Gender & Marital status & environment & education \\
\hline \multicolumn{5}{|l|}{ Stress } \\
\hline & $\begin{array}{l}T(68)= \\
-0.77\end{array}$ & $t(68)=0.35$ & $\begin{array}{l}t(65)=- \\
0.28\end{array}$ & $F(2,65)=0.95$ \\
\hline & $\begin{array}{l}p= \\
0.44\end{array}$ & $p=0.72$ & $p=0.77$ & $p=0.39$ \\
\hline \multirow[t]{4}{*}{$\begin{array}{l}\text { Quality } \\
\text { of life }\end{array}$} & $\begin{array}{l}t(68)= \\
0.51\end{array}$ & $t(68)=-0.62$ & $\begin{array}{l}t(65)=- \\
0.08\end{array}$ & $F(2,65)=0.01$ \\
\hline & $\begin{array}{l}p= \\
0.60\end{array}$ & $p=0.53$ & $p=0.93$ & $p=0.98$ \\
\hline & $\begin{array}{l}\mathrm{Mf}= \\
103.44\end{array}$ & $\mathrm{Ms}=47.8$ & $\mathrm{Mu}=103.83$ & $\begin{array}{l}M(<8 \text { years of } \\
\text { schooling) = } \\
104.02\end{array}$ \\
\hline & $\begin{array}{l}\mathrm{Mm}= \\
104.85\end{array}$ & $\mathrm{Mr}=51.63$ & $\mathrm{Mr}=51.43$ & $\begin{array}{l}M(9-12 \text { years of } \\
\text { schooling })= \\
104.02 \\
M(>12 \text { years of } \\
\text { schooling })= \\
104.00\end{array}$ \\
\hline
\end{tabular}

Likewise, no significant differences were obtained according to the demographic variables taken into account in this study, in what concerns hemodialysis patients' quality of life. These data are characteristic of the group of subjects that participated in this research and cannot be generalized.

\section{Discussions}

The level of stress has a significant effect on the quality of life, the physical health, but also the mental health of subjects who participated in this research. Certainly, these results cannot be generalized because of the small number of subjects and their very specific features. However, they represent a warning signal and underline the necessity for larger-scale studies, possibly for longitudinal studies to be carried out.

Hemodialysis patients undergo a particular risk of experiencing high-intensity stress in response to the confrontation with the overwhelming multitude of stressors associated with the chronic disease and the related treatment (Simmons, 1995; Gardikiotis, Popa, Manole \& lorga, 2015). Quality of life, as it is perceived by end-stage kidney disease patients, is known to be an important factor acting at the level of the results determined by the treatment of renal function replacement. Studies comparing hemodialysis patients' quality of life to that of the general population have noted the negative impact of the chronic disease and its associated treatment on patients' lives (Mohammad, 2006). 
Within our study, the dimension of physical symptoms associated with hemodialysis was designated, through the patients' answers, as the dimension which exerts the highest level of stress, consequently being the most problematic. Thus, the feeling of nausea and vomiting, in conjunction with the tension generated by muscle cramps, itchiness, sleep disorders, painful joints, fatigue and the loss of bodily functions place the dimension they make up in the first place of the hierarchy of stressors specific to hemodialysis.

In our study, food and fluid restrictions are considered the second most powerful stressome other studies obtained suprisingly results (Welch, Austin \& Frauman, 1999) proving that, for example, many of the 86 subjects who participated in a study declared they were not stressed at all by the limits imposed on fluid intake, because they did not comply with them. Some other studies (Mok \& Tam, 2001) on the other hand, obtained results according to which fluid restrictions are the most stressful aspect of hemodialytic treatment, followed by food restrictions. Taking into account that the economic situation of the subjects in our research group is poor to average, we may understand why a particular diet may be difficult to follow and stressful at the same time. What is more alarming is that hemodialysis patient who does not comply with food and fluid restrictions having an increased risk of mortality and a low life expectancy (John, Alpert, Kawi \& Tandy, 2013).

Functional vascular access is necessary in order for hemodialysis to be performed a fact which is particular to this chronic disease and to this population of patients. All these aspects place this stressor in the third position of our hierarchy.

In most cases, after dialysis sessions, patients are much too tired to engage in solving various recreational or household tasks. From an active person, the hemodialysis patient becomes an individual chained to his own health condition. Being in the presence of medical staff three times a week for approximately 4 hours, the hemodialysis patient may develop a relationship of dependence on them. In the present research, this stressor is located among the last three stress factors, more exactly, in the fifth position. Diagnosed with CKD, the individual acquires a new identity, that of a hemodialysis patient. What happens to the identity of a parent, a life partner, or to professional identity? The fact that the individual may become confused with regard to his role in the family or within society place the role ambiguity factor on the sixth place in our hierarchy.

It is highly possible that the recording of the lowest level of stress caused by the functioning of the reproductive system be due to the average age of the subjects included in this study $(M=54.55, D S=$ 11.70). Another explanation may be that women usually enter menopause around the age of 45-55 (Roberts, 2006) their interest in reproducing being significantly lower than among younger, fertile women.

In order to obtain a comprehensive image, we ranked the 31 items, which actually represent a particular stressor, according to the way in which they were assessed by the 70 subjects of this research.

The item assessed as a powerful stressor is that referring to the fatigue experienced by subjects. Fatigue drastically restricts the individual's ability to complete an activity, whether it is of an intellectual or recreational nature. Analyzing the table above, we may notice that most of the first 10 items identified as being the most stressful are of a physiological nature. We find one single item of a psychological nature, that is, "the uncertainty of the future". Fatigue was assessed as the most intense stressor in the study conducted by Harwood and collaborators (Harwood et al, 2009) the following positions being occupied by sleep disorders, peripheral neuropathy (numbness/prickling), muscle cramps, etc. Placing the item referring to the patient's role reversal with his own children in one of the last positions of the hierarchy of stressors is in accordance with the other results (Ahmad \& Nazly, 2015).

One of the important points of this research is that the authors of this study were interested in identifying both the level of stress caused by each individual stressor, represented by an item of the 
survey, and the level summarized by the totality of specific hemodialytic factors. This may foster the development of specific intervention strategies aimed at reducing the level of stress caused by a particular stressor, thus making it possible to ensure a high efficiency of the strategy and an improvement in the quality of life of the hemodialysis patient.

To identify the significant predictors of hemodialysis patients' quality of life, 7 simple linear regressions were performed. The results emphasize the fact that four of the seven predictors are significant for the studied criterion. Daily activity, dependence on medical staff, food and fluid restrictions and role ambiguity are negative predictors for hemodialysis patients' quality of life.

For the participants in this study, the satisfaction experienced in relation to the areas important to them decreases as the level of stress generated by daily activity, dependence on medical staff, food and fluid restrictions, as well as that generated by role ambiguity increases. These stressors represent important aspects of an individual's life, like those related to daily transport to the hemodialysis clinic, the uncertainty of the future, his relationship with medical staff, the diet and his role in the family or within society.

Physical health is one of the two dimensions of quality of life studied during this research. Because chronic kidney disease primarily debilitates patients' physical health status, the identification of its most important predictors is enlightening. By applying the method of multiple linear regression, we identified two significant predictors of the physical health criterion, namely, daily activity and dependence on medical staff. These two predictors are directly related to hemodialysis patients' health status. Again, the predictor with the most powerful effect on physical health is dependence on medical staff. The quality of the medical act significantly influences the progression of chronic kidney disease. When patients are satisfied with the way in which they are cared for and monitored both by nurses and by nephrologists, they may become stressed about the possibility of changes occurring in this respect. In addition, frequent hospitalization best explains the existence of poor physical health, or may predict poor physical health, implying the existence of major difficulties in carrying out activities such as climbing stairs, covering a certain distance on foot, reducing working time, reducing the nature of the work, easily falling ill, the worsening of one's health and, let us not forget, the pain experienced.

We may notice a vulnerability of mental health, in that it is affected by the level of stress patients experience in relation to several factors, compared to physical health. The more people undergoing hemodialysis as a form of treatment experience a high level of stress regarding their daily activity, physical symptoms, dependence on medical staff, food and fluid restrictions and role ambiguity, the more their mental health is negatively affected. Mental health represents, in fact, the patient's vitality, the extent to which he fulfills the social function, the valence of emotions experienced and the way in which these interfere with the patient's daily activities. Therefore, the more the level of stress related to the above-mentioned factors increases, the more the dimensions of mental health are affected, the patient gradually losing his vitality, becoming less and less active socially, experiencing more and more strongly emotions such as sadness, despondency, nervousness, which, in their turn, will prevent him from fulfilling his tasks in an efficient manner.

No significant differences have been identified between the level of stress experienced by subjects involved in a relationship and those who were single. It is possible for the result obtained to have been influenced by the disproportionality existing between the number of subjects involved in a relationship and that of single subjects.

The differences between the standard of living of people in rural areas and those in urban areas are significant, those in urban areas having a higher level of comfort. Hemodialysis patients who live in rural areas need transport to the hemodialysis center; they spend more time on the road and, in case of a medical emergency, they get to the hospital later than those in urban areas. However, no significant differences have been identified between the stress experienced by subjects depending on 
their original environment. This result may also be related to the placement of the stress generated by transport to/from the clinic in one of the last positions.

The most significant limitation of this scientific paper is that related to the impossibility of generalizing the results obtained. Taking into account the outstanding peculiarities of the subjects who were part of this research group, especially those connected to health status and its associated comorbidities, it would be unscientific to claim that the tendencies observed by us may be applicable to all patients diagnosed with CKD who undergo hemodialysis as a form of treatment. In addition, the transversal nature of the study limits the results obtained only to the moment when the assessment was performed and the data were collected by means of the instruments used. The small number of participants is another factor hindering the generalization of results, as well as the fact that these were selected within one single hemodialysis center. The intrusive character of the items and the delicate topic tackled could determine emotional and cognitive storms, this fact having significant implications on the results. Moreover, the manifestation of a facade trend may be assumed at the level of the subjects. Regardless of the aspect discussed, human individuals tend to outline a positive image in front of others, so the acknowledgement of one's own needs and afflictions could create the impression of vulnerability. Such a vulnerable image is hard to accept even by the individual himself, being difficult to reveal to another person. In the future, conducting longitudinal studies which take into account patients' progression from the start of the hemodialytic treatment for stage 5 chronic kidney disease over several years would offer the possibility of identifying the intensity of the effect of stressors on the same individuals over time.

As research about quality of life is still at an early stage in Romania, various themes can be explored, such as quality of life in family members of hemodialysis patients or in the medical staff in charge of providing the medical services.

Last, but not least, we could take into account the testing of various intervention strategies, in order to identify the best methods to improve certain aspects of a life that is less lived than measured, in days and years.

\section{Conclusions}

This research shows that, approached from this perspective, therapeutic intervention is effective in that the level of pain tolerance rises, thus decreasing the probability that participants harm themselves in future stress-generating situations. During the execution of the sentence, people deprived of liberty get used to being ignored, perceived as irretrievably compromised at a human level, with no real chance of changing and adopting a socially desirable conduct. When these suspicions are invalid, the need for self-disclosure, for requesting and accepting specialized help to solve personal problems, prevails. The results obtained confirm the connection between self-harming behavior and anxiety. Another aspect worth mentioning is methodology, the investigated number of subjects being relatively small. This is a common factor in clinical research, which does not allow a generalization of the obtained data, results being considered valid only for the investigated batch. To generalize, additional investigations are necessary, that is, implementing the intervention successively on several samples and comparing these, thus obtaining a higher degree of certainty about the results. At the level of this research, data are for guidance.

\section{References}

Ahmad, M. M., \& Nazly, E. K. (2015). Hemodialysis: Stressors and coping strategies. Psychology, health \& medicine, 20(4), 477-487. 
Covic, A., Seica, A., Gusbeth-Tatomir, P., Gavrilovici, O., \& Goldsmith, D. J. (2004). Illness representations and quality of life scores in haemodialysis patients. Nephrology Dialysis Transplantation, 19(8), 2078-2083.

Gardikiotis, I., Popa, M., Manole, A. M., \& Iorga, M. (2016). The Impact of Body Image and Self-Perceived Physical Ability on the Well-Being after Mastectomy without Reconstruction. Journal of Surgery, 2015

Harwood, L., Wilson, B., Locking-Cusolito, H., Sontrop, J., \& Spittal, J. (2009). Stressors and coping in individuals with chronic kidney disease.Nephrology Nursing Journal, 36(3), 265-301.

John, A., Alpert, P. T., Kawi, J., \& Tandy, R. (2013). The relationship between self-efficacy and fluid and dietary compliance in hemodialysis patients. Clinical Scholars Review, 6(2), 98-104.

Mohammad, N. A. E. G. (2006). Health related quality of life among Egyptian patients on hemodialysis. J. Med. Sci, 6(3), 314-320.

Mok, E., \& Tam, B. (2001). Stressors and coping methods among chronic haemodialysis patients in Hong Kong. Journal of clinical nursing, 10(4), 503-511.

Roberts, S. S. (2006). Living through menopause. Women go through many transitions in their lives, and diabetes affects how they experience a lot of them. Menopause--the time when periods stop--is no exception. Diabetes forecast, 59(5), 66.

Seica, A., Segall, L., Verzan, C., Vaduva, N., Madincea, M., Rusoiu, S., \& Grajdeanu, L. (2009). Factors affecting the quality of life of haemodialysis patients from Romania: a multicentric study. Nephrology Dialysis Transplantation, 24(2), 626-629.

Simmons, P. B. (1995). Predictors of quality of life in the chronic hemodialysis patient.

Welch, J. L., Austin, J. K., \& Frauman, A. C. (1999). Factors associated with treatment-related stressors in hemodialysis patients/Research critique/Investigator's response. Nephrology Nursing Journal, 26(3), 318. 